\title{
The Electronic Structure and Specific Heat of $\mathrm{YNi}_{4} \mathrm{Si}$
}

\author{
M. Pugaczowa-Michalska, M. Falkowski, A. KowalczyK \\ Institute of Molecular Physics, PAS \\ M. Smoluchowskiego 17, 60-179, Poznań, Poland \\ M. Timko, M. Reiffers, M. Mihalik
}

Institute of Experimental Physics, SAS, Watsonowa 38, 04353 Košice, Slovakia

\section{J. ŠEBeK AND E. ŠAnTAVA}

Institute of Experimental Physics, Academy of Sciences of the Czech Republic Na Slovance 2, 18221 Praha 8, Czech Republic

The studies of the electronic structure and the specific heat of $\mathrm{YNi}_{4} \mathrm{Si}$ are reported. Below the Fermi energy $\left(E_{\mathrm{F}}\right)$ the density of states contains mainly the $3 d$ states of $\mathrm{Ni}$, which hybridized with $4 d$ states of $\mathrm{Y}$ and $3 p$ states of Si. The theoretical electronic specific heat coefficient $\left(12.32 \mathrm{~mJ} /\left(\mathrm{mol} \mathrm{K}^{2}\right)\right)$ obtained for equilibrium lattice parameters and the experimental value $\left(13 \mathrm{~mJ} /\left(\mathrm{mol} \mathrm{K}^{2}\right)\right)$ are in a reasonable agreement.

PACS numbers: 71.20.Be, 71.20.-b, 65.40.-b

\section{Introduction}

In recent years ternary compounds $\mathrm{RNi}_{4} \mathrm{Si}$, containing the rare-earth metal $(\mathrm{R})$, have been intensively studied [1-3]. These compounds have a variety of interesting properties such as a mixed-valence state of the Ce ion, a Kondo-like resistivity impurity in $\mathrm{CeNi}_{4} \mathrm{Si}$ as well as a strong Mott-type scattering in resistance in $\mathrm{YbNi}_{4} \mathrm{Si}[2,3]$. From the electronic structure point of view these compounds are of special interest due to the nearly filled $\mathrm{Ni}(3 d)$ band implying that the Ni magnetic moment is negligible [1]. The $\mathrm{YNi}_{4} \mathrm{Si}$ compound is indeed useful considered as the isostructural non-magnetic reference material. Therefore, it is important to characterize its ground-state properties such as electronic structure from $a b$ initio method and specific-heat experiments, which give information on magnetic properties and energy level distribution. The $\mathrm{YNi}_{4} \mathrm{Si}$ belongs to a wide 
class of compounds which crystallise in the hexagonal $\mathrm{CaCu}_{5}$ structure (space group $P 6 / \mathrm{mmm}$ ). In the $\mathrm{CaCu}_{5}$ structure the $\mathrm{Ni}$ atoms occupy the crystallographic sites $(2 \mathrm{c})$ and $(3 \mathrm{~g})$, while the rare-earth atoms are located in (1a) and $\mathrm{Si}$ atoms occupy $(3 \mathrm{~g})$ sites.

\section{Experimental details and calculation method}

The electronic structure was calculated by using the tight-binding linear muffin-tin orbital (TB LMTO) method in the atomic sphere approximation (ASA) [4]. Details of calculation were the same as that for $\mathrm{CeNi}_{4} \mathrm{Si}$ [1]. The sample preparation procedure for $\mathrm{YNi}_{4} \mathrm{Si}$ is similar to that for $\mathrm{CeNi}_{4} \mathrm{Si}$ [1]. The lattice constants are $a=4.79 \AA$ and $c=4.692 \AA\left(\mathrm{YNi}_{4} \mathrm{Si}\right)$. Specific heat measurements were performed by PPMS commercial device (Quantum Design) in the temperature range $4-300 \mathrm{~K}$ by relaxation method using two- $\tau$ model. The error of the measurement was about $2 \%$.

\section{Results}

The electronic structure calculations for $\mathrm{YNi}_{4} \mathrm{Si}$ have shown that its ground state is paramagnetic. The calculated densities of states (DOS) are shown in Fig. 1a. The DOS reflects a large separation of the low-lying bands and the main part of DOS (from -0.497 Ry to $E_{\mathrm{F}}$ ) with respect to the corresponding band widths. Si $3 s$ band is located deeply at the lower part of DOS [ -0.73 Ry; $-0.58 \mathrm{Ry}$ ] below the $E_{\mathrm{F}}$. In the upper part of the DOS (above $-0.497 \mathrm{Ry}$ ) $3 d$
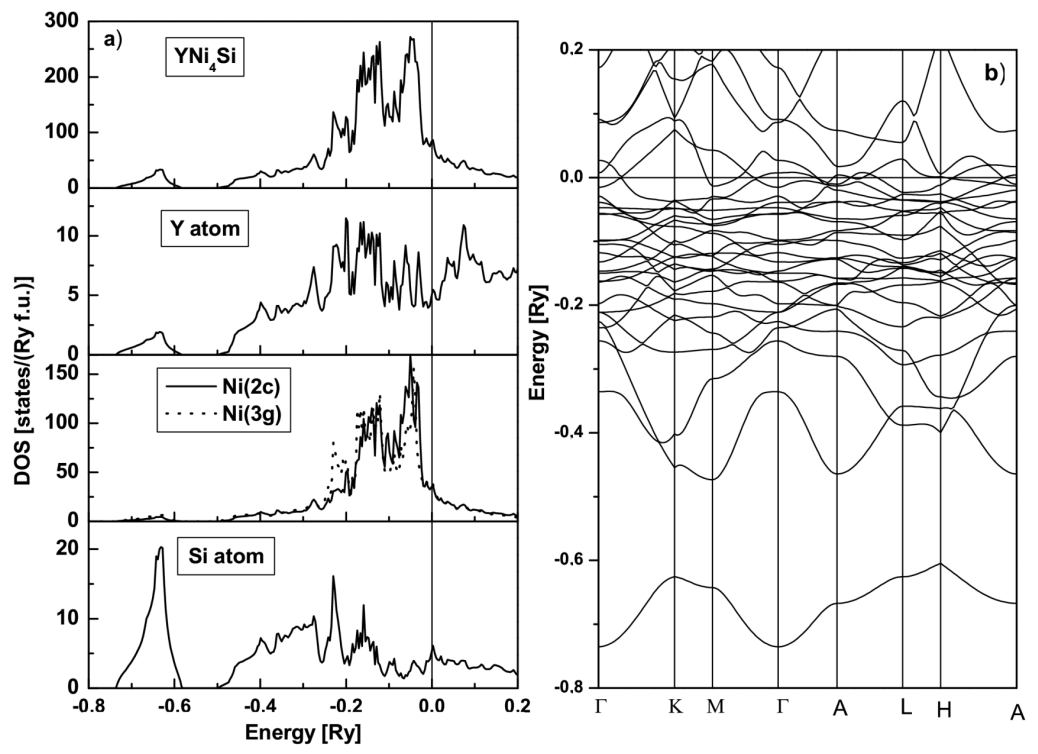

Fig. 1. (a) The calculated DOS for $\mathrm{YNi}_{4} \mathrm{Si}$ : the total DOS; the contribution of $\mathrm{Y}$; the contribution of $\mathrm{Ni}(2 \mathrm{c})$ and $\mathrm{Ni}(3 \mathrm{~g})$; the contribution of $\mathrm{Si}$. (b) The band structure of $\mathrm{YNi}_{4} \mathrm{Si}$ along the high symmetry directions. 
Ni states overlap with $4 d \mathrm{Y}$ states. The hybridization of Si $3 p, \mathrm{Y} 4 d$ and $\mathrm{Ni} 3 d$ levels results in continuous DOS from the energy of -0.5 Ry to the Fermi energy. The band structure of $\mathrm{YNi}_{4} \mathrm{Si}$ along selected high-symmetry lines within the first Brillouin zone (BZ) is shown in Fig. 1b. The shape of the lowest band is strikingly similar to that of $\mathrm{CeNi}_{4} \mathrm{Si}$ [1]. The bottom of the lowest band of $\mathrm{YNi}_{4} \mathrm{Si}$, which consists entirely Si $3 s$ states, has the parabolic shape around the $\Gamma$ point of high symmetry in the $\Gamma-A, \Gamma-M, \Gamma-K$ direction (below $-0.7 \mathrm{Ry}$ ) as well as in the $A-H$ direction (below $-0.65 \mathrm{Ry}$ ). The group of bands formed between $-0.5 \mathrm{Ry}$ and the $E_{\mathrm{F}}$ is mainly dominated by Ni $3 d$ states mixed with $\mathrm{Si} 3 p$ states. $E_{\mathrm{F}}$ is crossed in all main directions of the first BZ by the bands. The $K, L, \Gamma, M$ points of high symmetry bands do not cross $E_{\mathrm{F}}$. The DOS at $E_{\mathrm{F}}$ is 85.55 [st./(Ry f.u.)] for $\mathrm{YNi}_{4} \mathrm{Si}$. The electronic specific heat coefficient can be estimated from band calculations by using the relation:

$$
\gamma=\frac{1}{3} \pi^{2} k_{\mathrm{B}}^{2} N\left(E_{\mathrm{F}}\right)
$$

and is equal to $14.8 \mathrm{~mJ} /\left(\mathrm{mol} \mathrm{K}^{2}\right)$.

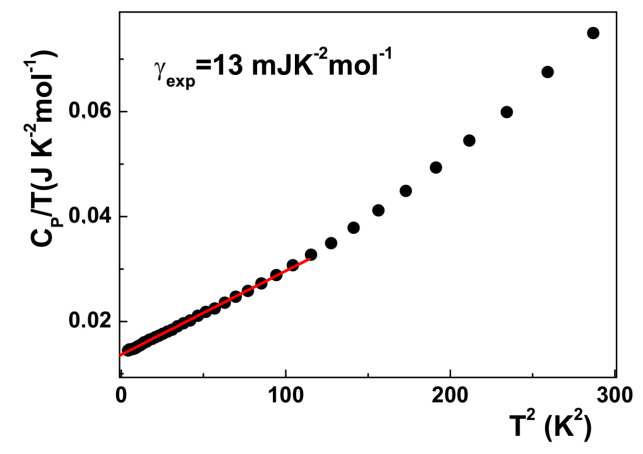

Fig. 2. Specific heat of $\mathrm{YNi}_{4} \mathrm{Si}$, plotted as $C_{p} / T$ versus $T^{2}$.

The temperature dependence of heat capacity $C_{p}$ measured for $\mathrm{YNi}_{4} \mathrm{Si}$ is presented in Fig. 2. The total heat capacity consists of two contributions: the electronic specific heat $C_{\mathrm{el}}$ characterized by the Sommerfield coefficient $\gamma$ and the phonon contribution $C_{\mathrm{ph}}$ which can be expressed as:

$$
C_{p}=C_{\mathrm{el}}+C_{\mathrm{ph}}=\gamma T+\beta T^{3} .
$$

The Debye temperature is estimated using the relation:

$$
\Theta_{\mathrm{D}}=\left(\frac{12 \pi^{4} R n}{5 \beta}\right)^{\frac{1}{3}}
$$

where $n$ is a number of atom in the unit cell, $R$ is the gas constant. The experimental values are: $\gamma=13 \mathrm{~mJ} /\left(\mathrm{mol} \mathrm{K}{ }^{2}\right) ; \Theta_{\mathrm{D}}=388 \mathrm{~K}$. The value of $\gamma$ obtained from electronic structure for experimental lattice parameters is somewhat higher than the experimental $\gamma$. However, minimization procedure of the total energy 
of $\mathrm{YNi}_{4} \mathrm{Si}$ gives the equilibrium lattice constants of $a=4.551 \AA, c=4.41 \AA$ and value of $\gamma=12.32 \mathrm{~mJ} /\left(\mathrm{mol} \mathrm{K}^{2}\right)$. Thus, the experimental value and the theoretical value of the electronic specific heat coefficient obtained at equilibrium lattice parameters are in good agreement. Moreover, the theoretical values of $\gamma$ obtained for $\mathrm{YNi}_{4} \mathrm{Si}$ in this work are close to the value of $11.33 \mathrm{~mJ} /\left(\mathrm{mol} \mathrm{K}^{2}\right)$ determined for $\mathrm{YNi}_{4} \mathrm{~B}[6]$ and $13.42 \mathrm{~mJ} /\left(\mathrm{mol} \mathrm{K}^{2}\right)$ determined for $\mathrm{YNi}_{4} \mathrm{Cu}[5]$.

\section{Conclusions}

$A b$ initio calculation has shown that the $\mathrm{YNi}_{4} \mathrm{Si}$ is paramagnetic. Below the $E_{\mathrm{F}}$ the total DOS contained mainly Ni $3 d$ states in $(2 \mathrm{c})$ and $(3 \mathrm{~g})$ positions hybridized with Y $4 d$ states and Si $3 p$ states which formed the main part of the valence band. The theoretical electronic specific heat coefficient is in good agreement with that obtained from experiments.

\section{Acknowledgments}

This work was supported partly by the COST-ECOM P16, by Science and Technology Assistance Agency APVT-51-031704, by VEGA6165; by the contract CE of SAS. J.S. and E.S. are grateful for support of GACR106/06/0368.

\section{References}

[1] A. Kowalczyk, M. Falkowski, V.H. Tran, M. Pugaczowa-Michalska, J. Alloys Comp. 440, 13 (2007).

[2] A. Kowalczyk, M. Falkowski, T. Toliński, G. Chełkowska, Solid State Commun. 139, 5 (2006).

[3] A. Kowalczyk, M. Falkowski, T. Toliński, V.H. Tran, W. Miiller, M. Reiffers, M. Timko, Mater. Res. Bull. 43, 185 (2008).

[4] O.K. Andersen, O. Jepsen, M. Sob, in: Electronic Structure and Its Applications, Ed. M.S. Yussouff, Springer, Berlin 1987, p. 2.

[5] M. Pugaczowa-Michalska, G. Chełkowska, A. Kowalczyk, Acta Phys. Pol. A 104, 487 (2003).

[6] T. Toliński, M. Pugaczowa-Michalska, G. Chełkowska, A. Kowalczyk, J. Electron. Spectrosc. Relat. Phenom. 151, 1 (2006). 